\title{
Path-Following Algorithms and Experiments for an Unmanned Surface Vehicle
}

\author{
Marco Bibuli \\ Istituto di Studi sui Sistemi Intelligenti per l'Automazione \\ Consiglio Nazionale delle Ricerche \\ Via De Marini 6, 16149 Genova, Italy \\ marcolge.issia.cnr.it \\ Gabriele Bruzzone \\ Istituto di Studi sui Sistemi Intelligenti per l'Automazione \\ Consiglio Nazionale delle Ricerche \\ Via De Marini 6, 16149 Genova, Italy \\ gabryage.issia.cnr.it \\ Massimo Caccia \\ Istituto di Studi sui Sistemi Intelligenti per l'Automazione \\ Consiglio Nazionale delle Ricerche \\ Via De Marini 6, 16149 Genova, Italy \\ max@ge.issia.cnr.it

\section{Lionel Lapierre} \\ Laboratoire d'Informatique, de Robotique et de Microlectronique de Montpellier \\ Centre National de la Recherche Scientifique \\ 161 rue Ada, 34392 Montpellier Cedex 5, France \\ lapierredirmm. fr
}

\begin{abstract}
This paper addresses the problem of path-following in two-dimensional space for underactuated unmanned surface vehicles (USVs), defining a set of guidance laws at kinematic level. The proposed nonlinear Lyapunov-based control law yields convergence of the path following error coordinates to zero. Furthermore, the introduction of a virtual controlled degree of freedom for the target to be followed on the path removes singularity behaviors present in other guidance algorithms proposed in the literature. Some heuristic approaches are then proposed to face the problem of speed of advance adaptation based on path curvature measurement and steering action prediction. Finally a set of experimental results of all the proposed guidance laws, carried out with the Charlie USV, demonstrate the feasibility of the proposed approach and the performance improvements, in terms of precision in following the reference path and transient reduction, obtained introducing speed adaptation
\end{abstract}


heuristics.

\section{Introduction}

In the last fifteen years a large number of Unmanned Surface Vehicles (USVs) have been developed for a large set of applications such as environmental monitoring and sampling, coastal protection, bathymetric surveys, support for Autonomous Underwater Vehicles (AUVs) operations.

Some examples are given by the family of USVs at the MIT AUV Lab (Manley et al., 2000) (Benjamin and Curcio, 2004); the flotilla of autonomous marine vehicles, such as Delfim and Caravela, at the Lisbon IST-ISR Dynamical System and Ocean Robotics Laboratory (Pascoal and et al., 2000) (Alves et al., 1999); the Charlie USV originally developed by CNR-ISSIA Genova for sea surface microlayer sampling and then applied to restricted water operations (Caccia et al., 2007); the ROAZ autonomous surface vehicles developed by the Instituto Superior de Engenharia do Porto also devoted to search and rescue support (Martins et al., 2006); and the catamaran Springer developed by the Plymouth University to monitor and track water pollution (Xu et al., 2006).

On the military side, the project SWIMS, i.e. shallow water influence mine sweeping system, by QinetiQ Ltd successfully demonstrated a conversion kit able to convert standard RIBs (rigid inflatable boats) in remotely controlled ones, during the second Gulf War (Cornfield and Young, 2006). After that, research mainly focuses on the development and integration of sensors for overthe-water obstacle detection and avoidance. Examples are given by the testbed developed at SSC San Diego (Ebken et al., 2005), based on the Bombardier SeaDoo Challenger 2000, and the Israeli Protector USV (Pro, ), equipped with radar and advanced electro-optical devices.

For major details about USV technology, the reader can refer to (Caccia, 2006) and (Manley, 2008). In this context, the existing prototype USVs, typically not equipped with side thrusters and thus under-actuated, are required to perform tasks which need increasing manoeuvring accuracy, moving, for instance, from the goal of executing an integral sampling in a relatively large area to the aim of reconstructing a very precise bathymetry of a littoral zone.

Generally speaking, the problems related to motion control of UMVs are classified in the literature into three basic groups:

- point stabilization: the goal is to stabilize the vehicle zeroing the position and orientation error with respect to a given target point, with a desired orientation. The goal cannot be achieved with smooth or continuous state-feedback control laws, when the vehicle has nonholonomic constraints; in this case, approaches like smooth time-varying control laws and discontinuous and hybrid feedback laws have been proposed;

- trajectory tracking: the vehicle is required to track a time-parameterized reference. For fully actuated system, the problem can be solved with advanced nonlinear control laws; in the case of under-actuated vehicles, that is, the vehicle has less degrees of freedom than state variables to be tracked, the problem is still a very active topic of research;

- path following: the vehicle is required to converge to and follow a path, without any temporal specification. The assumption made in this case is that the vehicle's forward speed tracks a desired speed profile, while the controller acts on the vehicle orientation to drive it to the path. This typically allows a smoother convergence to the desired path with respect to the trajectory tracking controllers, less likely pushing to saturation the control signals (Encarnaçao and Pascoal, 2001). 
Since the requirement of following desired paths with great accuracy with a speed profile specified by the end-user is sufficient for many applications, the problem of path-following, i.e. steering a vehicle to converge to and follow a predefined path in the plane, is addressed in this paper.

The path-following problem, originally addressed in the literature in the case of wheeled robots, consists in defining, computing and reducing to zero the distance between the vehicle and the path as well as the angle between the vector representing the vessel speed and the tangent to the desired path. In the case of unmanned marine vehicles, a solution based on gain-scheduling control theory and the linearization of a generalized error vector about trimming paths has been proposed in (Pascoal et al., 2006) and implemented and run on the Delfim Autonomous Surface Craft. After that, research focused on the development of nonlinear control design methods able to guarantee global, and not only local, stability as in the above-mentioned approach.

In particular, research in this direction has been guided by the key idea of controlling the rate of progression of a "virtual target", also named rabbit, that has to be tracked, thus bypassing the problem of singularities, that can arise when the target is defined as the simple projection of the real vehicle on the path. The original formulation for wheeled ground robots can be found in (Lapierre et al., 2003), while its application to autonomous underwater vehicles (AUVs), combined with backstepping control design methodologies, is presented in (Lapierre and Soetanto, 2006). A preliminary experimental validation for USVs has been presented in (Bibuli et al., 2007). On the other hand, the approach introduced in (Indiveri et al., 2007), and experimentally validated with a testbed USV in (Bibuli et al., 2008), explicitly addressed the underactuation of the vehicle already when defining the error variable to be globally and robustly stabilized to zero. Anyway, path follower performances can be enhanced by using preview controller design techniques as introduced in (Gomes et al., 2006). The role of the guidance system, computing all the reference signals needed to make the physical system autonomous, as well as the need of developing the guidance theory at the kinematic level in order to make it as general as possible, are discussed in (Breivik and Fossen, 2004). That work proposes a parameter adaptation technique to introduce integral action for environmental disturbance compensation.

According to the requirement of designing a generic path-following system, the application to a small autonomous catamaran, the Charlie USV, will be discussed in the sequel, pointing out the integration with the vehicle navigation and control system and the design and implementation of heuristics able to increase performances on the basis of experimental results. In particular, a kinematic guidance law, generating a proper yaw-rate reference signal to drive the vehicle above the path, is combined with an already implemented PI-type velocity control level. On the other hand, some heuristic laws are introduced to face the problem of surge speed adaptation in function of the path curvature and steering action prediction. The surge speed reference value is modulated in a range of preset values in order to speed up the convergence to the required heading and to maintain the vehicle on the path when the path curvature increases.

The paper is organized as it follows: in section 2 the vehicle kinematics is described, both in free space and referred to the path-following task. A brief overview of an operational model of the Charlie USV dynamics is presented too. Section 3 discusses the proposed path-following guidance algorithms together its relations with the system navigation, guidance and control architecture, and basic implementation issues. A couple of heuristic speed adaptation laws able to increase system performance are introduced too. The Charlie USV is presented in section 4, while experimental results are reported and discussed in section 5. 


\section{Modeling}

\subsection{General kinematics}

Assuming that the vessel motion is restricted to the horizontal plane, i.e. neglecting pitch and roll, two reference frames are considered: an inertial, earth-fixed frame $\langle e\rangle$, where position and orientation $\left[\begin{array}{ll}x y & z\end{array}\right]^{T}$ of the vessel are usually expressed, and a body-fixed frame $\langle b\rangle$, where surge and sway velocities ( $[u v]^{T}$ absolute, $\left[u_{r} v_{r}\right]^{T}$ with respect to the water), yaw rate $r$ and forces and moments $\left[\begin{array}{lll}X & Y & N\end{array}\right]^{T}$ are represented.

Denoting with $\left[\dot{x}_{C} \dot{y}_{C}\right]^{T}$ the sea current, supposed to be irrotational and constant, i.e. $\ddot{x}_{C}=\ddot{y}_{C}=0$, the vehicle kinematics is usually modelled with the following equations, which are expressed in the earth-fixed frame:

$$
\left\{\begin{array}{l}
\dot{x}=u_{r} \cos \psi-v_{r} \sin \psi+\dot{x}_{C} \\
\dot{y}=u_{r} \sin \psi+v_{r} \cos \psi+\dot{y}_{C} \\
\dot{\psi}=r
\end{array}\right.
$$

Assuming that the vessel is moving at constant surge with respect to the water with negligible sway, i.e. $v_{r}=0$ and $\dot{u}_{r}=\dot{v}_{r}=0$, as in the case of the Charlie USV, the kinematic model (1) can be rewritten in terms of the total velocity as it follows:

$$
\left\{\begin{array}{l}
\dot{x}=U \cos \psi_{e} \\
\dot{y}=U \sin \psi_{e} \\
\dot{\psi}_{e}=r\left[\frac{u_{r}^{2}}{U^{2}}+\frac{u_{r}}{U^{2}}\left(\dot{x}_{C} \cos \psi+\dot{y}_{C} \sin \psi\right)\right]=r \eta(t)
\end{array}\right.
$$

where

$$
\begin{aligned}
U & =\sqrt{\dot{x}^{2}+\dot{y}^{2}} \\
\psi_{e} & =\arctan \frac{\dot{y}}{\dot{x}}
\end{aligned}
$$

denote the module and orientation of the vehicle speed in the earth-fixed reference frame $\langle e\rangle$. It is worth noting that the rotation rate of the vehicle speed orientation in the frame $\langle e\rangle$ is function of a time variable parameter $\eta(t)$, inducted by sea currents, as shown by the $3^{\text {rd }}$ equation of (2).

A graphical representation of the nomenclature of the USV kinematics is given in Figure 1, where variables describing the path-following problem are pointed out too.

\subsection{Path-following kinematics}

Given the path to be followed by the vehicle, a Serret-Frenet frame $<f>$ that moves along the path is defined. Such frame is usually called virtual target vehicle and it should be tracked by the real vehicle. With reference to Figure $1, P$ is an arbitrary point on the path, $\langle f\rangle$ is the SerretFrenet frame associated to that point and $\mathbf{p}=\left[\begin{array}{ll}x_{P} & y_{P}\end{array}\right]^{T}$ is the position vector of the point $P$ with reference to the earth fixed frame $\langle e\rangle$. The point $B$, attached to the vehicle body, can either be expressed as $\mathbf{x}=\left[\begin{array}{lll}x & y & 0\end{array}\right]^{T}$ in $\langle e\rangle$ or as $\left[\begin{array}{lll}s_{1} & y_{1} & 0\end{array}\right]^{T}$ in $\langle f\rangle$.

The rotation matrix from $\left\langle e>\right.$ to $\langle f\rangle$, parameterized locally by the angle $\psi_{f}$, which is the 


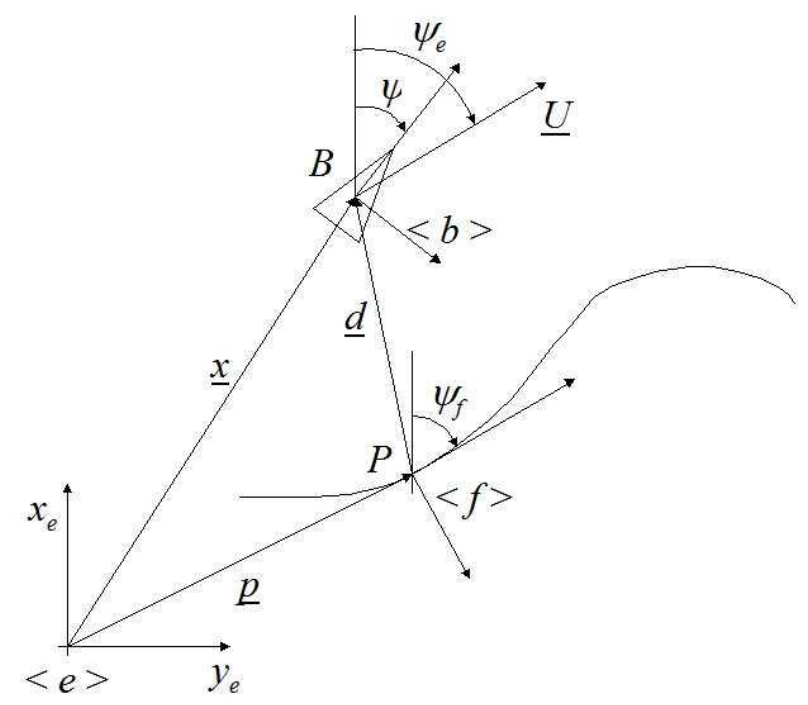

Figure 1: Vehicle's parameters and frames definition

tangent to the path at point $P$, is denoted by

$$
R=\left[\begin{array}{ccc}
\cos \psi_{f} & \sin \psi_{f} & 0 \\
-\sin \psi_{f} & \cos \psi_{f} & 0 \\
0 & 0 & 1
\end{array}\right]
$$

Defining $r_{f}=\dot{\psi}_{f}$, and denoting with $s$ the signed curvilinear abscissa along the path, the following expressions hold

$$
\left\{\begin{array}{c}
r_{f}=\dot{\psi}_{f}=c_{c}(s) \dot{s} \\
\dot{c}_{c}(s)=g_{c}(s) \dot{s}
\end{array}\right.
$$

where $c_{c}(s)$ and $g_{c}(s)=\frac{d c_{c}(s)}{d s}$ denote the path curvature and its derivative, respectively.

The velocity of $P$ in the Serret-Frenet frame $\langle f>$ and the velocity of $B$ in earth-fixed frame $<e>$ are respectively:

$$
\left(\frac{d \mathbf{p}}{d t}\right)_{f}=\left[\begin{array}{l}
\dot{s} \\
0 \\
0
\end{array}\right]
$$

and

$$
\left(\frac{d \mathbf{x}}{d t}\right)_{e}=\left(\frac{d \mathbf{p}}{d t}\right)_{e}+R^{-1}\left(\frac{d \mathbf{d}}{d t}\right)_{f}+R^{-1}\left(\left[\begin{array}{lll}
0 & 0 & r_{f}
\end{array}\right]^{T} \times \mathbf{d}\right)
$$

where $\mathbf{d}$ is the vector from $P$ to $B$.

The velocity of $B$ in $\langle e\rangle$ expressed in $\langle f\rangle$, obtained pre-multiplying the above equation by $R$, is

$$
\left(\frac{d \mathbf{x}}{d t}\right)_{f}=R\left(\frac{d \mathbf{x}}{d t}\right)_{e}=\left(\frac{d \mathbf{p}}{d t}\right)_{f}+\left(\frac{d \mathbf{d}}{d t}\right)_{f}+\left[\begin{array}{lll}
0 & 0 & r_{f}
\end{array}\right]^{T} \times \mathbf{d}
$$

Using the relations

$$
\left(\frac{d \mathbf{x}}{d t}\right)_{e}=\left[\begin{array}{l}
\dot{x} \\
\dot{y} \\
0
\end{array}\right]
$$




$$
\left(\frac{d \mathbf{d}}{d t}\right)_{f}=\left[\begin{array}{c}
\dot{s}_{1} \\
\dot{y}_{1} \\
0
\end{array}\right]
$$

and

equation (2) can be rewritten as

$$
\left[\begin{array}{lll}
0 & 0 & r_{f}
\end{array}\right]^{T} \times \mathbf{d}=\left[\begin{array}{c}
0 \\
0 \\
c_{c}(s) \dot{s}
\end{array}\right] \times\left[\begin{array}{c}
s_{1} \\
y_{1} \\
0
\end{array}\right]=\left[\begin{array}{c}
-c_{c}(s) \dot{s} y_{1} \\
c_{c}(s) \dot{s} s_{1} \\
0
\end{array}\right]
$$

$$
R\left[\begin{array}{c}
\dot{x} \\
\dot{y} \\
0
\end{array}\right]=\left[\begin{array}{c}
\dot{s}\left(1-c_{c}(s) y_{1}\right)+\dot{s}_{1} \\
\dot{y}_{1}+c_{c}(s) \dot{s} s_{1} \\
0
\end{array}\right]
$$

Solving for $\dot{s}_{1}$ and $\dot{y}_{1}$ yields

$$
\left\{\begin{array}{l}
\dot{s}_{1}=\left[\begin{array}{ll}
\cos \psi_{f} & \sin \psi_{f}
\end{array}\right]\left[\begin{array}{l}
\dot{x} \\
\dot{y}
\end{array}\right]-\dot{s}\left(1-c_{c} y_{1}\right) \\
\dot{y}_{1}=\left[\begin{array}{ll}
-\sin \psi_{f} & \cos \psi_{f}
\end{array}\right]\left[\begin{array}{l}
\dot{x} \\
\dot{y}
\end{array}\right]-c_{c} \dot{s} s_{1}
\end{array}\right.
$$

Finally, replacing the top two equations of (2) in (3) and introducing the variable $\beta=\psi_{e}-\psi_{f}$ gives the 'kinematic' model of the vehicle in $(s, y)$ coordinates as

$$
\left\{\begin{array}{l}
\dot{s}_{1}=-\dot{s}\left(1-c_{c} y_{1}\right)+U \cos \beta \\
\dot{y}_{1}=-c_{c} \dot{s} s_{1}+U \sin \beta \\
\dot{\beta}=r_{e}-c_{c} \dot{s}
\end{array}\right.
$$

where $r_{e}=\dot{\psi}_{e}=r \eta(t)$.

\subsection{Dynamics}

As mentioned in section 1, the work presented in this paper focuses on the design of a pathfollowing guidance law at kinematic level, while the generated reference yaw rate is tracked by a low-level dedicated controller already present on the testbed USV. In particular, the Charlie USV is equipped with model-based linear and angular velocity controllers, as well as motion estimators. Thus, a brief discussion of the adopted model of the vehicle dynamics is reported in the following. For a detailed discussion of the assumptions and experimental results, which led to the definition of a practical model ${ }^{1}$ for the Charlie USV dynamics, the reader can refer to (Caccia et al., 2008b). In particular, since the vessel speed with respect to the water is about proportional to the propeller revolution rate, experiments carried out with the Charlie USV revealed the impossibility to observe both these quantities, as well as sway dynamics, using measurements only from onboard GPS and compass. Thus, sway speed can be neglected and the dynamics can be reduced to:

$$
\begin{aligned}
\tilde{m}_{u} \dot{u}_{r} & =\tilde{k}_{u} u_{r}+\tilde{k}_{u_{r}^{2}} u_{r}^{2}+\tilde{k}_{n^{2} \delta^{2}} n^{2} \delta^{2}+n^{2} \\
\tilde{I}_{r} \dot{r} & =\tilde{k}_{r} r+\tilde{k}_{r|r|} r|r|+\tilde{k}_{n^{2}} n^{2}+n^{2} \delta
\end{aligned}
$$

\footnotetext{
${ }^{1}$ practical stands for consistent, from the point of view of degree of accuracy, quality in terms of noise and sampling rate of the measurements
} 
where $n$ is the propeller revolution rate, $\delta$ is the rudder angle, $\tilde{m}_{u}$ and $\tilde{I}_{r}$ are the normalised inertia terms, $\tilde{k}_{u}, \tilde{k}_{u_{r}^{2}}, \tilde{k}_{r}$ and $\tilde{k}_{r|r|}$ are the drag coefficients, $\tilde{k}_{n^{2} \delta^{2}}$ represents the resistance due to the rudder and $\tilde{k}_{n}^{2}$ takes into account the vessel longitudinal asymmetries.

Since, as discussed above, in equation (5), the steering torque $n^{2} \delta$ has been identified as function of the propeller revolution rate instead of the advance speed, the rudder action is neglected when the vehicle is still moving while $n$ is zero. Thus, it is worth noting that the field of validity of the proposed model of vehicle dynamics is for $n>\bar{n}>0$. As remarked in section 3.3, this forces a minimum USV speed for guaranteeing manoeuvring capabilities.

\section{Guidance and control}

\subsection{Navigation, guidance and control architecture}

A dual-loop hierarchical guidance and control architecture decoupling kinematics and dynamics is adopted. In the proposed control scheme, the external guidance loop performs position control generating suitable velocity references according to the desired task, i.e. path-following in this case. The dynamic controllers have to ensure that the actual rotational and linear speed of the vehicle track the references with sufficient precision to guarantee the overall stability of the system. Although a rigorous demonstration of system stability is not given, the design of guidance task functions at the kinematic level is usually very simple as well as the tuning of the kinematic and dynamic controller parameters on the basis of empirical considerations as discussed in (Caccia, 2007) and (Caccia et al., 2008a).

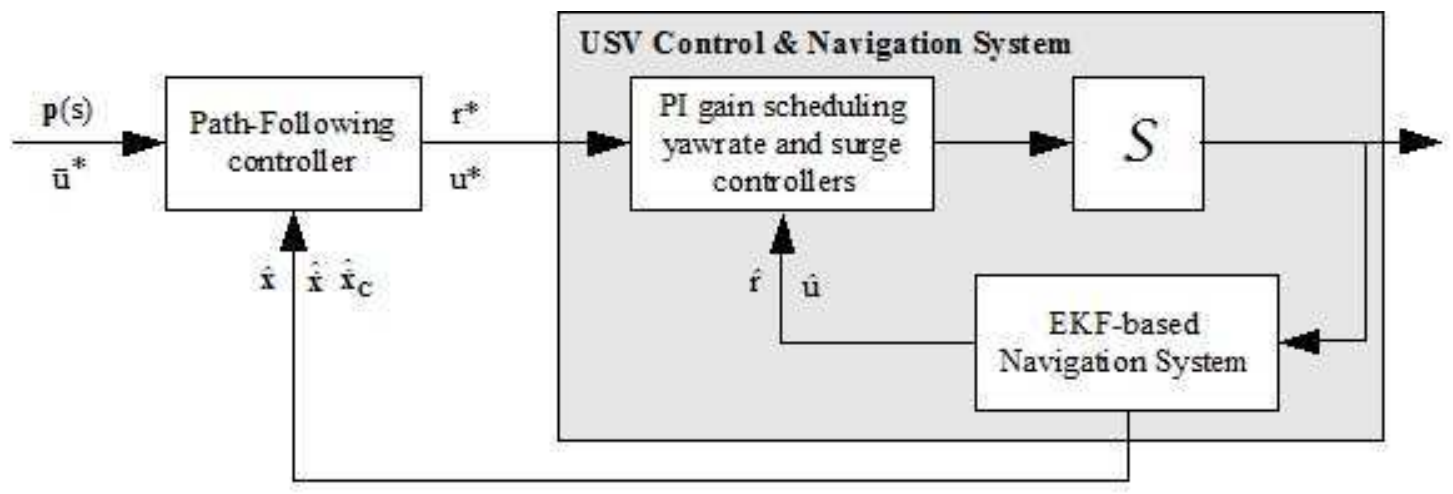

Figure 2: Dual-loop navigation, guidance and control architecture

As shown in Figure 2, this architecture implements different motion task functions keeping unchanged the vehicle control and navigation system. In the case of the Charlie USV, a navigation system relying on model based extended Kalman filters estimates the vessel position $\mathbf{x}$, orientation and speed $\dot{\mathbf{x}}$, as well sea current velocity $\dot{\mathbf{x}}_{C}$, in the earth-fixed frame $\langle e\rangle$ and surge $u$ and yaw rate $r$ in the body-fixed frame $\langle b\rangle$. PI-type linear and angular velocity controllers are designed following a gain scheduling approach in order to guarantee a specific behaviour, in terms of closed-loop characteristic equations. A detailed discussion of the Charlie USV navigation and control system can be found in (Caccia et al., 2008a). Here, it is sufficient to point out that the kinematic path-following guidance law, computed as discussed in section 3.2, feeds a lower 
level PI-type yaw-rate dynamic controller. Although the proof of stability of the overall dual-loop control scheme is not considered in this paper, the kinematic controller parameters are assumed to be set such that the rate of change of $r^{*}$ is slow enough to be perfectly tracked by the dynamic controller.

\subsection{Kinematic controller design}

Usually, the solution to the path-following problem is based on the zeroing of the distances between the vehicle and a point $P$ on the path, and the angle $\beta$ between the vehicle's total velocity vector $U$ and the tangent to the path at $P$. With respect to classical approaches in which $P$ is the closest point on the path, the key idea proposed in this paper is to consider the target point, with its associated Serret-Frenet frame $\langle f\rangle$, moving along the path according to a defined control law, obtaining in this way an extra controller design parameter.

As discussed in (Lapierre and Soetanto, 2006), the demonstration of stability of the proposed controller is essentially based on the application of the Barbalat's lemma and LaSalle's theorem.

Since the application of LaSalles theorem is restricted to autonomous systems, in the examined case the fact that the desired forward velocity is a constant allows the system to be considered as autonomous. In the following, a trace of the main steps in designing the Lyapunov based controller is given. For a rigorous proof the reader can refer to (Lapierre and Soetanto, 2006).

At first a desired approach angle $\varphi$ is defined as a function of the distance of the USV from the tangent line to the path in the point $P$, i.e. $y_{1}$ in the Serret-Frenet frame $\left\langle f>\right.$. The function $\varphi\left(y_{1}\right)$ has to satisfy the constraints $\left\|\varphi\left(y_{1}\right)\right\|<\pi / 2, y_{1} \varphi\left(y_{1}\right) \leq 0$ and $\varphi(0)=0$, stating that the vehicle heads for the desired path and remains over it once reached.

For instance, the following hyperbolic tangent shaped function, parameterized by $k_{\varphi}>0$ and $0<\psi_{a}<\pi / 2$, with its saturation properties satisfies the above mentioned requirements:

$$
\varphi\left(y_{1}\right)=-\psi_{a} \tanh \left(k_{\varphi} y_{1}\right)
$$

Then, the vehicle approach angle $\beta$ is imposed to track the desired one $\varphi$ by considering the candidate Lyapunov function

Its time derivative

$$
V=\frac{1}{2}(\beta-\varphi)^{2}
$$

$$
\dot{V}=(\dot{\beta}-\dot{\varphi})(\beta-\varphi)=\left(r \eta(t)-c_{c} \dot{s}-\dot{\varphi}\right)(\beta-\varphi)
$$

is negative-semidefinite, $\dot{V}=-k_{1}(\beta-\varphi)^{2}$, when choosing the control law

$$
r^{*}=\frac{1}{\eta(t)}\left[\dot{\varphi}-k_{1}(\beta-\varphi)+c_{c}(s) \dot{s}\right]
$$

where $k_{1} \geq 0$.

Since $V$ is lower bounded and $\dot{V}$ is uniformly continuous, Barbalat's lemma leads to the conclusion that $\lim _{t \rightarrow+\infty} \dot{V}=0$.

Moreover, the proposed control law makes variables $\beta, s_{1}$ and $y_{1}$ bounded and approaching to the set $E$, defined by $\dot{V}=0$. The motion of the feedback control system, restricted to $E$ can be studied considering the Lyapunov function

Computing its time derivative

$$
V_{E}=\frac{1}{2}\left(s_{1}^{2}+y_{1}^{2}\right)
$$

$$
\dot{V}_{E}=(U \cos \beta-\dot{s}) s_{1}+U y_{1} \sin \beta
$$


it is worth noting that the speed $\dot{s}$ of the target Serret-Frenet $\langle f\rangle$ constitutes an additional degree of freedom that can be controlled in order to guarantee the convergence of the vehicle at the desired path. In particular, considering that, in the set $E, \beta=\varphi\left(y_{1}\right)$ and that $y_{1} \sin \varphi\left(y_{1}\right) \leq 0$ for the choice of $\varphi\left(y_{1}\right)$, the control law

$$
\dot{s}^{*}=U \cos \beta+k_{2} s_{1}
$$

with $k_{2}>0$ guarantees $\dot{V}_{E} \leq 0$. Since $\ddot{V}_{E}$ is bounded, for Barbalat's Lemma $\lim _{t \rightarrow+\infty} \dot{V}_{E}=0$, which in turn implies that all trajectories in $E$ satisfy $\lim _{t \rightarrow+\infty} s_{1}=0$ and $\lim _{t \rightarrow+\infty} y_{1}=0$. Thus the asymptotically convergence to zero of the variables $\beta, s_{1}$ and $y_{1}$ is guaranteed.

The controller exposed in equation (7) with the virtual target equation of motion (8) has been implemented on the Charlie USV's architecture. Experimental results are reported in section 5.

The advantage of the proposed controller are summarised as it follows.

- The nonlinear approach angle $\varphi$, see equation (6), originally introduced by C. Samson (Micaelli and Samson, 1993) allows for driving the incidence angle of the robot with respect to path. Indeed when $y_{1}$ is high, the desired incidence is $\pm \psi_{a}$, i.e. $\pm \frac{\pi}{2}$, that is the vehicle's orientation is driven perpendicularly to the path tangent. As $y_{1}$ reduces, the incidence also reduces, and vanishes to zero as $y_{1}$ is zero, i.e. when the vehicle is on the path.

- The virtual target principle, denoted as $\dot{s}$, introduces an extra (and virtual) degree of freedom to the system. Controlling the virtual target as expressed in equation (8) removes the classic singularity of the path-following problem exposed in (Micaelli and Samson, 1993). Indeed, the consideration of the closest point on the path as the target to be tracked by the vehicle imposes a severe limitation in the domain of attraction of the control. The virtual target allows for enlarging this domain of attraction to the whole space, thus removing singularity.

\subsubsection{Implementation issues}

\section{- Sea current compensation}

As discussed in (Caccia et al., 2008a), where the case of straight line following has been addressed, lateral sea currents are naturally compensated by guidance laws generating a reference yaw rate as a function only of the range from the target path and its first derivative. Indeed, thanks to an integrator embedded in the kinematics of the linearized physical system around the equilibrium point, the vehicle naturally heads in such a way of following the desired line while compensating lateral sea current. This property, which guarantees local stability, is not valid when the reference yaw rate is somehow computed as a function of a desired heading angle. In this case, the presence of neglected constant sea current disturbances yields to a steady state error in the range from the desired path.

Since the measurement of sea current is not always available onboard small USVs, and its estimate can be very imprecise or noisy, operating conditions can require the adoption of guidance laws neglecting this class of disturbances. How neglecting sea current affects the proposed path-following guidance law is discussed in the following.

In the equation (7) the term $\eta(t)=\left[\frac{u_{r}^{2}}{U^{2}}+\frac{u_{r}}{U^{2}}\left(\dot{x}_{c} \cos \psi+\dot{y}_{c} \sin \psi\right)\right]$ can be unknown, or roughly estimated. Anyway, adding some practical constraints the multiplicative noise term could be neglected. Indeed, considering the typical operative conditions in which the 
vehicle surge with respect to the water is higher than the sea current

$$
\sqrt{\dot{x}_{C}^{2}+\dot{y}_{C}^{2}}<u_{r}
$$

the noise function $\eta(t)$ is always positive and never reaches the zero value.

So, assuming $\eta(t)=1$, i.e. neglecting sea currents, and rewriting the guidance law for $r$ as

$$
r^{*}=\dot{\varphi}-k_{1}(\beta-\varphi)+c_{c}(s) \dot{s}
$$

the derivation of $V$ yields:

$$
\dot{V}=-k_{1} \eta(t)(\beta-\varphi)^{2}+(\eta-1)\left(\dot{\varphi}+c_{c} \dot{s}\right)(\beta-\varphi)
$$

that is negative outside of the interval limited by zero and $\frac{\eta(t)-1}{k_{1} \eta(t)}\left(\dot{\varphi}+c_{c} \dot{s}\right)$, that defines a tube around the path that the system is guaranteed to reach.

\section{- Behaviour at path beginning and end}

The desired path has typically a beginning and an end, i.e. is typically defined for constrained values of the curvilinear abscissa. Thus, the evolution of $s$ according to (8) has to be constrained in the interval $s \in\left[0 s_{M A X}\right]$. In particular, forcing $s$ to 0 or $s_{M A X}$ when its evolution would lead it outside of the desired interval is equivalent to force the vehicle to follow the tangent to the path in its beginning or end point respectively. From a practical point of view, this is usually not dangerous when the vehicle approaches the path starting point along its tangent. On the other hand, a vehicle which tries to move along the path tangent after its end can be very dangerous. So, safety manoeuvres, e.g. rotating at constant yaw rate, have to be executed when the vehicle reaches the end of the path, i.e. $s^{*}=s_{M A X}$.

\section{- Generic path software representation}

The issue of generic representation of paths in the software implementation has been addressed too. Although only explicit representations through mathematical functions can guarantee exact computation of the path properties for every value of the curvilinear abscissa $s$, from the point of view of software implementation such an approach would limit the possible paths to a set of classes of functions known by the system. Thus, a generic path is represented as a sequence of points, discretised for $s=s_{0}, \ldots, s_{N}=s_{M A X}$, with the corresponding tangent vector and curvature. For intermediate values of $s$, the path parameters are linearly interpolated: $p(s)=p\left(s_{i}\right)+\frac{p_{\left.\left(s_{i+1}\right)-p_{(} s_{i}\right)}}{s_{i+1}-s_{i}}\left(s-s_{i}\right)$ for $s \in\left(s_{i}, s_{i+1}\right)$ where $p$ can represent the path point coordinates, tangent vector components or curvature.

\subsection{Heuristic speed adaptation}

The above discussed guidance law drives an ideal vessel with no leeway with respect to the water over a desired path. Indeed, the actual testbed USV is characterized by physical limitations on the maximum yaw rate and unmodeled sway with respect to the water. The result is that the vehicle, when working at constant surge, can execute large U-turns for heading the path or sliding away from the path when executing sharp curves.

The proposed solution to this issue arises from human common behavior when driving a vehicle: while approaching a curve or when tricky manoeuvres are needed, advance speed is reduced with respect to the desired one $\bar{u}^{*}$ established by the human operator or mission controller.

Assuming that the vehicle speed is within a minimum $U_{\text {min }}$, needed to allow manoeuvring capabilities (see section 2.3 for details), and a maximum $U_{\text {max }}$, a heuristic law has been introduced in 
the global regulation schema to improve the guidance and control system performances.

From this concept arises the equations that model such a behavior; first, an adaption for the maximum surge speed is computed in function of the actual yaw rate requested by the controller:

$$
u_{1_{M A X}^{*}}^{*}=\frac{U_{\max }-U_{\min }}{2}+\frac{U_{\max }-U_{\min }}{2}\left[\cos \left(\frac{\pi r^{*}}{r_{\text {sat }}}\right)\right]
$$

where $U_{\max }, U_{\min }$ and $r_{\text {sat }}$ are parameters of the adaptation law. This reduces surge speed when the vehicle orientation is far from the required heading in order to speed up the convergence to the desired approach angle. Thus, a second adapted maximum reference surge speed is computed on the basis of a prediction of the maximum curvature of the path inside a predefined prediction horizon:

$$
u_{2_{M A X}}^{*}=U_{\min }+\left(U_{\max }-U_{\min }\right)\left[1-\tanh ^{2}\left(k_{u} c_{\max }\right)\right]
$$

where $k_{u}$ is a free parameter and $c_{\max }$ is the maximum value of $c_{c}(\bar{s})$, with $\bar{s}$ in $[s ; s+h], h$ is the prediction horizon. This helps the vehicle to maintain its position above high curvature segments of the path. Finally, the adapted maximum reference surge speed is computed as the minimum between $u_{1_{M A X}}^{*}$ and $u_{2_{M A X}^{*}}^{*}$ :

$$
u_{M A X}^{*}=\min \left(u_{1_{M A X}^{*}}^{*}, u_{2_{M A X}^{*}}^{*}\right)
$$

and the adapted reference surge is

$$
u^{*}=\min \left(\bar{u}^{*}, u_{M A X}^{*}\right)
$$

The reader should note that the expression (9) contains the control input $r^{*}$, thus introducing a coupling between the yaw and surge control inputs. The authors are aware of the algebraic loop introduced by this method. Nevertheless, note that this coupling will induce a reduced surge velocity. The improvement of this method is clearly exposed in section 5, where experimental results validated the proposed approach.

\section{Charlie USV}

The Charlie USV (see Figure 3) is a small catamaran-like shaped prototype vehicle, originally developed and exploited, during the XIX Italian expedition to Antarctica in 2003-'04, by the CNRISSIA for the sampling of the sea surface microlayer and immediate subsurface for the study of the sea-air interaction (Caccia et al., 2005). Charlie is $2.40 \mathrm{~m}$ long, $1.70 \mathrm{~m}$ wide and weighs about $300 \mathrm{Kg}$ in air. The propulsion system of the vehicle is composed by a couple of DC motors (300 W @ $48 \mathrm{~V}$ ), with a set of servo-amplifiers which provide a proportional-integral-differential (PID) control of the propeller revolution rates. In the current release, the vehicle is equipped with a rudder-based steering system, where two rigidly connected rudders, positioned behind the thrusters, are actuated by a brushless DC motor. The navigation instrumentation set is constituted by a GPS Ashtech GG24C integrated with a KVH Azimuth Gyrotrac able to compute the True North. Electrical power supply is provided by four $12 \mathrm{~V} @ 40 \mathrm{Ah}$ lead batteries integrated with four $32 \mathrm{~W}$ triple junction flexible solar panels. The on-board real-time control system, developed in $\mathrm{C}++$, is based on GNU/Linux and run on a Single Board Computer (SBC), which supports serial and Ethernet communications and PC-104 modules for digital and analog I/O (Bruzzone et al., 2008). An overview of the Charlie project, including a detailed description of the vehicle and a summary of its applications, can be found in (Caccia et al., 2007). Nominal values for 


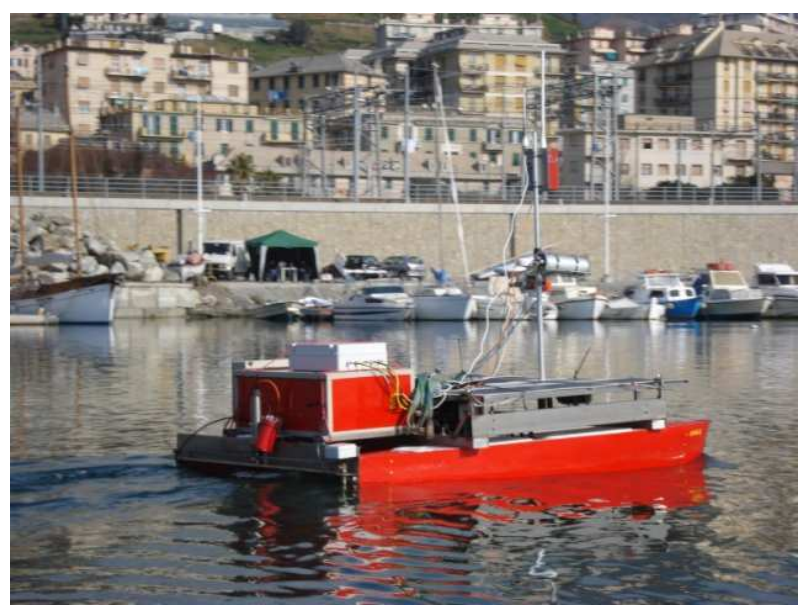

Figure 3: The Charlie USV

the parameters of the Charlie USV dynamics, as modelled in equations 4 and 5, can be found in (Caccia et al., 2008b).

\section{Experimental results}

Experimental tests have been carried out in the Genova Prà harbor (see Figure 4, a calm water channel devoted to rowing races; the site is usually beaten by a 20-30 knots wind. Results obtained during a preliminary session of trials, carried out in December 2006, are reported and discussed in (Bibuli et al., 2007), where the integration with backstepping techniques for handling the vehicle dynamics has been considered too. The tests discussed in the following have been performed on December $22^{\text {nd }} 2008$ in no wind conditions and on March $x x^{\text {th }} 2009$ in bla bla conditions. Field

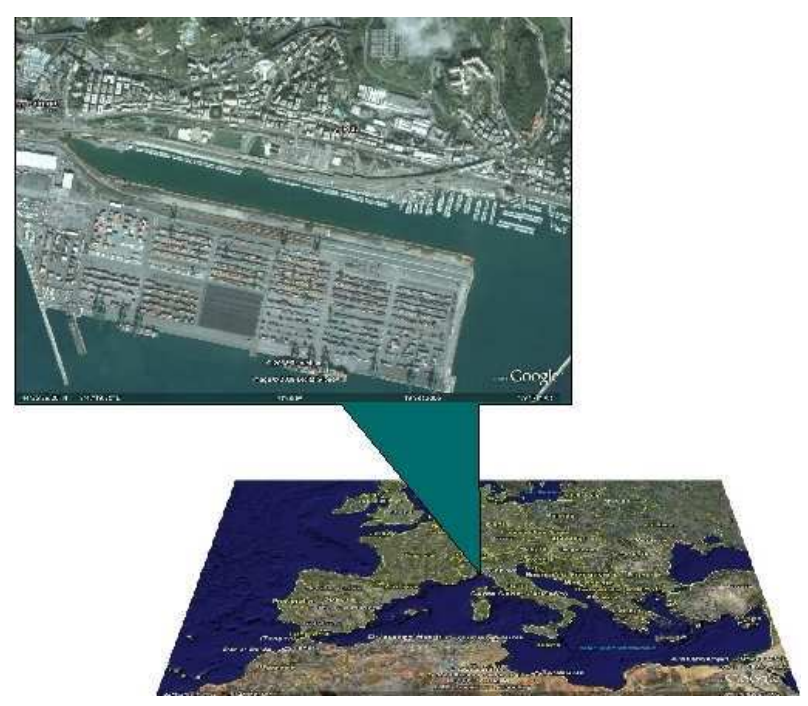

Figure 4: Genova Prà test site (Google Earth view)

trials had two basic goals: $i$ ) proving the practical validity of the proposed theoretical guidance laws 
(7) and (8); ii) evaluating the benfits given by heuristic speed adaptation. A set of metrics have been defined for measuring the main performance criterium, i.e. the capability of the vehicle of following the desired path: the area between the actual and the reference path measures the integral performance along the path, the bla bla. In this context, the kinematic guidance algorithm, with different approach angles, has been tested without speed adaptation and with the guidance law integrated with the speed adaptation heuristic, accomplishing dynamic control with the Charlie USV standard PI gain-scheduling yaw-rate controller. The vehicle navigation system consisted of conventional extended and linear time-varying Kalman filters processing GPS and compass measurements. Experimental identification of the vehicle dynamics allowed the open-loop estimate of its surge speed with respect to the water, on the basis of the normalised propeller revolution rate and rudder deflection by online integrating equation (4). This operation is equivalent to implement a kind of virtual velocity sensor consisting of an open-loop model-based predictor in the manner presented in (Caccia and Veruggio, 1999). For a detailed discussion of the Charlie USV navigation and control system the reader can refer to (Caccia et al., 2008a). The desired path was specified through a suitable graphical user interface, which allowed the operator to supervise the vehicle behaviour during the tests (see Figure 5). At first the capability of the proposed algorithm to drive

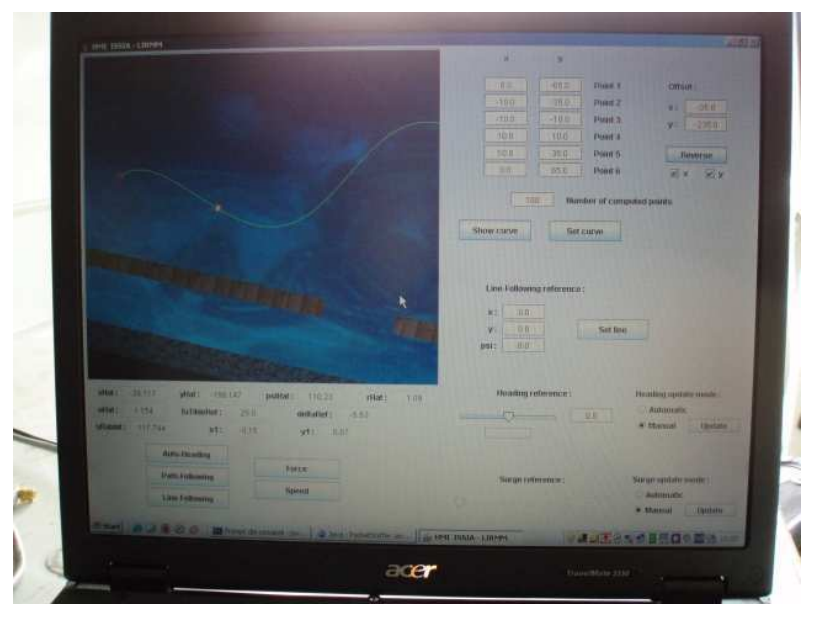

Figure 5: Charlie graphical user interface while monitoring path-following

the vehicle along a desired smooth path, i.e. with maximum curvature equal to about $0.032 \mathrm{~m}^{-1}$, has been demonstrated. As in all the other experiments presented in this paper, the USV reference surge was set to $1 \mathrm{~m} / \mathrm{s}$, while its minimum value was fixed at $0.6 \mathrm{~m} / \mathrm{s}$ in order to guarantee a smooth manoeuvrability of the vehicle. The hyperbolic tangent shaped approach angle $\varphi$ was parameterised by the values $\psi_{a}=60 \mathrm{deg}$ and $k_{\varphi}=0.3$, and the gains $k_{1}$ and $k_{2}$ the steering and virtual target speed control laws were assumed equal to 0.2 and 1.0 respectively. The reference path, as well as the vehicle motion with and without speed adaptation, is depicted in Figure 6. As shown in Figures 7 and 8, where the variables characterising the USV motion and error in following the desired path are plotted, the range $y_{1}$ from the path in the Serret-Frenet frame $\langle f\rangle$ is lower than $1.5 \mathrm{~m}$ in both the experiments. The USV diverges slightly from the path while turning basically because of the delay in copying the reference angular speed. Indeed, although reducing the surge speed while approaching a curve mainly according to the speed adaptation rule (10) with a preview horizon of $15 \mathrm{~m}$, the vehicle does not anticipate the turning action, thus not counteracting the effects induced by yaw rate dynamics. The reduction of the reference surge down to about 0.8 $\mathrm{m} / \mathrm{s}$ is clearly visible in the second plot of Figure 8. 


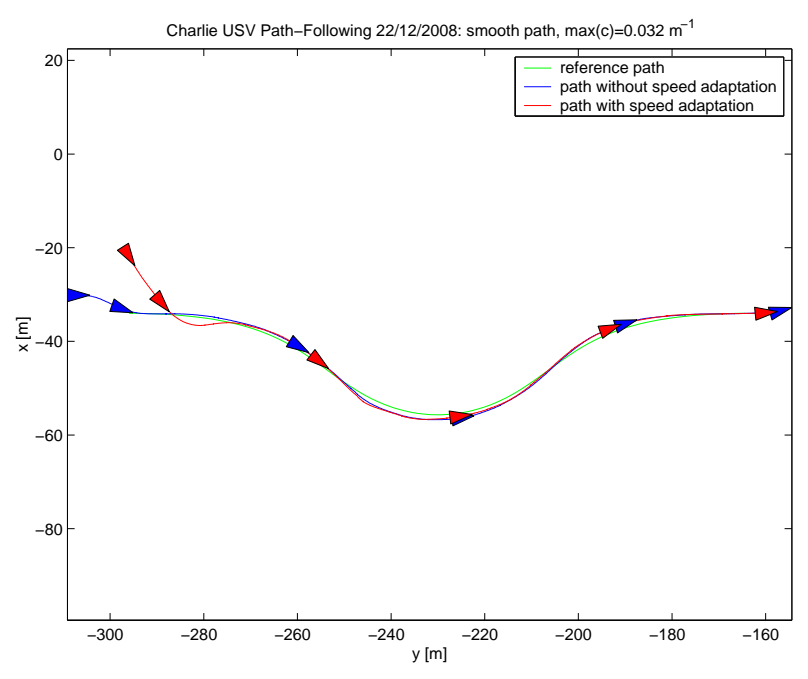

Figure 6: Path-Following: smooth path. Charlie USV estimated and desired path.

On the other hand, the model-based tuning of the yaw rate controller (Caccia et al., 2008a) allows a quite smooth action of the rudder actuator minimising the mechanical stress of the system.

The benefits given by speed adaptation while following high curvature paths and inverting the motion direction are pointed out by the second set of manoeuvres where the USV is requested to follow curves of similar shape of the previous one, but with a maximum curvature of $0.063 \mathrm{~m}^{-1}$. The two paths were shifted in the x-y plane due to the traffic conditions inside the regatta field where trials were performed. In this case, as clearly visible in Figure 9, the reduction of speed while approaching a curve, given by the speed adaptation rule (10), keeps the vehicle closer to the desired path moving along high curvature bents. Moreover, when reducing its surge while turning according to the speed adaptation rule (9), the vehicle executes U-turns in a smaller area recovering the desired path without remarkable overshoots induced by the combination of its constrained yaw rate and high reference surge speed.

Figures 10 and 11, where the guidance and navigation variables of the USV are plotted, provide some quantitative measurements of the benefits obtained by heuristically adapting the vehicle speed. The adaptation of the surge speed down to $0.6 \mathrm{~m} / \mathrm{s}$ reduces the range $y_{1}$ from the path in the Serret-Frenet frame $<f>$ to about $1.2 \mathrm{~m}$ with respect to more than $2 \mathrm{~m}$ when no speed adaptation rule is applied. In addition, the overshoot when re-tracking the desired path in the opposite direction decreases from more than $7 \mathrm{~m}$ to about $1 \mathrm{~m}$, while the approximate diameter of the U-turn decreases from about $20 \mathrm{~m}$ to less than $8 \mathrm{~m}$. It is worth noting that the apparent higher use of the rudder when speed adaptation is applied is due by the fact that, according to the USV yaw dynamics (5), at lower surge, i.e. at lower propeller revolution rate $n$, higher rudder angles are required for obtaining the same yaw rate.

The benefits given by adapting the surge speed in function of the actual yaw rate requested by the controller are pointed out when the Charlie USV followed a straight line in alternate directions as shown in Figures 12 and 13. In particular, the reduction of the USV surge speed with its yaw rate according to equation (9) dramatically restricts the circle-like path drawn by the vehicle while executing a U-turn. Indeed, with a default reference surge of $1 \mathrm{~m} / \mathrm{s}$, Charlie followed a path of about $22.5 \mathrm{~m}$ of diameter when no speed adaptation was applied, which reduced to about $10 \mathrm{~m}$ in the case of heuristic adaptation of the reference surge. The overshoot and oscillations, once reached 


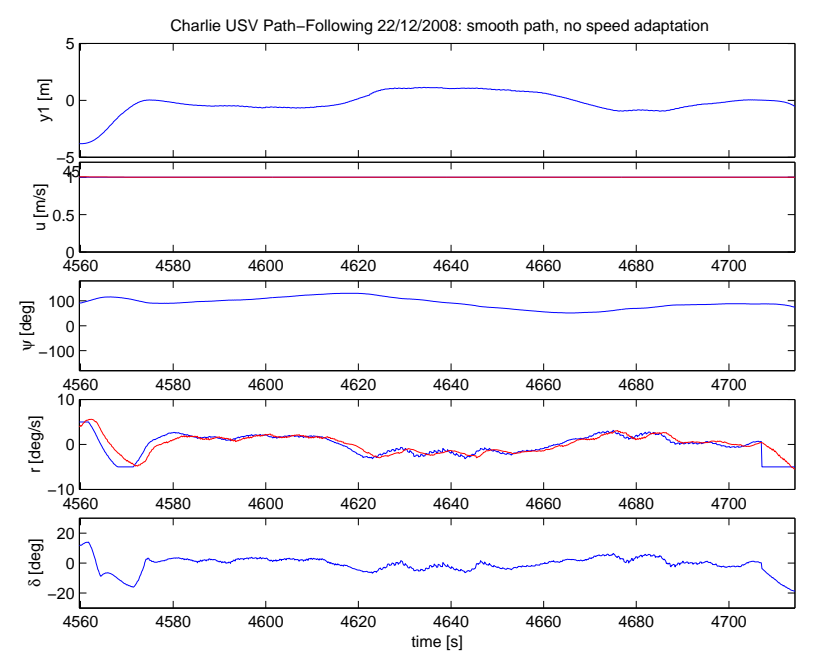

Figure 7: Path-Following: smooth path without speed adaptation. Charlie USV path-following and navigation variables. From top to bottom: range from the desired path $y_{1}$, reference and estimated surge $u^{*}$ and $\hat{u}$, estimated heading $\hat{\psi}$, reference and estimated yaw rate $r^{*}$ and $\hat{r}$, rudder angle $\delta$

the target path, reduced in a similar way. Moreover, the proposed guidance law demonstrated its capability in managing inversions of direction in following a line without any singularity.

\section{Conclusions}

In this paper the problem of path-following in two-dimensional space for under-actuated unmanned surface vehicles has been handled through the definition of a nonlinear Lyapunov-based guidance law, yielding convergence of the path following error coordinates to zero. Singularities of the algorithm are removed thanks to the introduction of the target dynamic. The proposed solution, which generates reference surge and yaw rate, has been integrated with the control system of an existing USV managing its dynamics in a conventional nested-loop architecture. Although a rigorous demonstration of system stability is not given, the design of the path-following guidance task at the kinematic level has been validated in extended field trials carried out with the Charlie USV, developed by CNR-ISSIA and employed as a testbed for the evaluation of numerous guidance and control techniques (Caccia et al., 2007).

Moreover, experimental results confirmed the expected improvements of the tracking response of the proposed technique obtained with the integration of the guidance law with some heuristic approaches, facing the problem of speed of advance adaptation based on path curvature measurement and steering action prediction. Although these heuristic approaches introduce an algebraic loop through a coupling between the yaw and surge control inputs, dramatic benefits in terms of tracking precision and execution of human-like manoeuvres have been verified experimentally.

Although experimental results show a manoeuvering precision of the order of a few tenths of centimeters, which is generically satisfactory for underactuated marine systems, a metric-based comparison with other guidance laws for path-following proposed in the literature would allow a quantitative evaluation of system performances. The implementation and integration in the vehicle control system of a set of path-following controllers, as well as the execution of comparative trials for straight line and generic path following, is part of the Charlie USV basic research plan. 

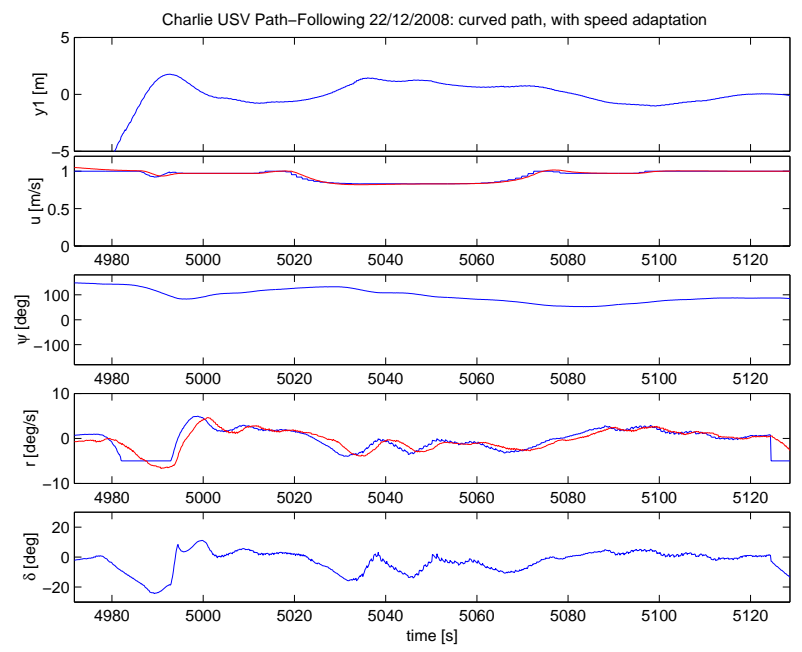

Figure 8: Path-Following: smooth path with speed adaptation. Charlie USV path-following and navigation variables. From top to bottom: range from the desired path $y_{1}$, reference and estimated surge $u^{*}$ and $\hat{u}$, estimated heading $\hat{\psi}$, reference and estimated yaw rate $r^{*}$ and $\hat{r}$, rudder angle $\delta$

Moreover, future works head towards the mathematical formalization of the heuristic laws and the demonstration of stability of the nested loop guidance and control system.

\section{Acknowledgments}

This work has been funded by the common research project "Sensor-based guidance and control of autonomous marine vehicles: path-following and obstacle avoidance" between CNR-ISSIA Genova, Italy, and CNRS-LIRMM Montpellier, France, for the years 2006-2007, and by PRAI-FESR within the project: "Coastal and harbor underwater anti-intrusion system" for the years 2005-2007. The authors wish to thank Giorgio Bruzzone and Edoardo Spirandelli for their fundamental support in developing, maintaining and operating the Charlie USV. Special thanks to the members of A.D.P.S. Prà Sapello for their kind support to sea trials.

\section{References}

Protector - Unmanned Naval Patrol Vehicle. http://www.israeliweapons.com/weapons/naval/protector/Protector.html.

Alves, T., Pascoal, A., Pereira, A., Rodeia, J., simoes, A., Juliano, M., Duarte, R., Silvestre, C., Oliveira, P., Sebastiao, L., Jorge, A., and Araujo, R. (1999). The use of CARAVELA 2000 vehicles in operational oceanography. In Proc. of 2nd Eurogoos Conference 1999.

Benjamin, M. and Curcio, J. (2004). COLREGS-based navigation in Unmanned Marine Vehicles. In IEEE Proceedings of AUV-2004.

Bibuli, M., Bruzzone, G., Caccia, M., Indiveri, G., and Zizzari, A. (2008). Line following guidance control: Application to the charlie unmanned surface vehicle. In Proc. of IEEE/RSJ 2008 International Conference on Intelligent RObots and Systems. 


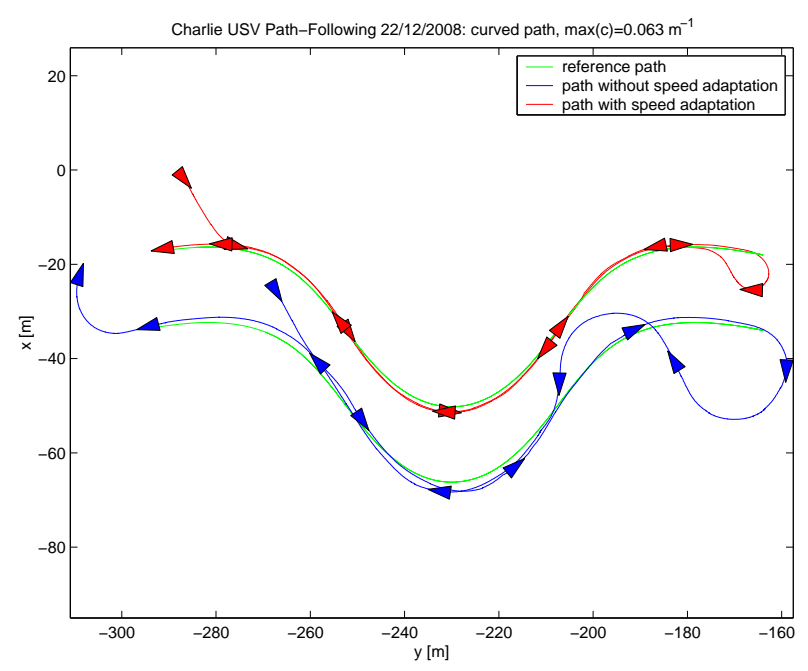

Figure 9: Path-Following: curved path. Charlie USV estimated and desired path.

Bibuli, M., Caccia, M., and Lapierre, L. (2007). Path-following algorithms and experiments for an autonomous surface vehicle. In Proc. of IFAC Conference on Control Applications in Marine Systems.

Breivik, M. and Fossen, T. (2004). Path following for marine surface vessels. In Proc. of OTO'04, pages 2282-2289.

Bruzzone, G., , Caccia, M., Bertone, A., and Ravera, G. (2008). Standard Linux for embedded real-time robotics and manufacturing control systems. Robotics and Computer Integrated Manufacturing. doi: 10.1016/j.rcim.2007.07.016.

Caccia, M. (2006). Autonomous Surface Craft: prototypes and basic research issues. In Proc. of IEEE 14th Mediterranean Conference on Control and Automation.

Caccia, M. (2007). Vision-based ROV horizontal motion control: near-seafloor experimental results. Control Engineering Practice, 15(6):703-714.

Caccia, M., Bibuli, M., Bono, R., and Bruzzone, G. (2008a). Basic navigation, guidance and control of an Unmanned Surface Vehicle. Autonomous Robots, 25(4):349-365.

Caccia, M., Bibuli, M., Bono, R., Bruzzone, G., Bruzzone, G., and Spirandelli, E. (2007). Unmanned Surface Vehicle for coastal and protected water applications: the Charlie project. $M a$ rine Technology Socety Journal, 41(2):62-71.

Caccia, M., Bono, R., Bruzzone, G., Bruzzone, G., Spirandelli, E., Veruggio, G., Stortini, A., and Capodaglio, G. (2005). Sampling sea surface with SESAMO. IEEE Robotics and Automation Magazine, 12(3):95-105.

Caccia, M., Bruzzone, G., and Bono, R. (2008b). A practical approach to modeling and identification of small autonomous surface craft. IEEE Journal of Oceanic Engineering. DOI: 10.1109/JOE.2008.920157.

Caccia, M. and Veruggio, G. (1999). Model-based heave motion estimation for variable configuration unmanned underwater vehicles. In Proc. of IFAC World Congress, Beijing, China.

Cornfield, S. and Young, J. (2006). Advances in unmanned marine vehicles, chapter Unmanned 


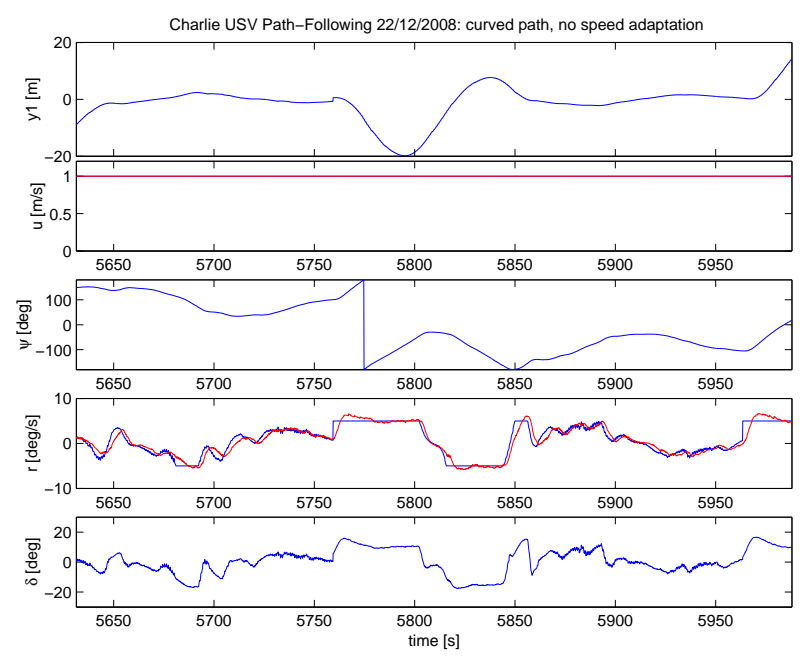

Figure 10: Path-Following: curved path without speed adaptation. Charlie USV path-following and navigation variables. From top to bottom: range from the desired path $y_{1}$, reference and estimated surge $u^{*}$ and $\hat{u}$, estimated heading $\hat{\psi}$, reference and estimated yaw rate $r^{*}$ and $\hat{r}$, rudder angle $\delta$

surface vehicles - game changing technology for naval operations, pages 311-328. IEE Control Series.

Ebken, J., Bruch, M., and Lum, J. (2005). Applying UGV technologies to unmanned surface vessel's. In SPIE Proc. 5804, Unmanned Ground Vehicle Technology VII.

Encarnaçao, P. and Pascoal, A. (2001). Combined trajectory tracking and path following: an application to the coordinated control of autonomous marine craft. In Proc. of 40th IEEE Conference on Decision and Control, volume 1, pages 964-969.

Gomes, P., Silvestre, C., Pascoal, A., and Cunha, R. (2006). A path-following controller for the DELFIMx autonomous surface craft. In Proc. of 7th IFAC Conference on Manoeuvring and Control of Marine Craft, Lisbon, Portugal.

Indiveri, G., Zizzari, A., and Mazzotta, V. (2007). Linear path following guidance control for underactuated ocean vehicles. In Proc. of 2007 IFAC Conference on Control Applications in Marine Systems.

Lapierre, L. and Soetanto, D. (2006). Nonlinear path following control of an auv. Accepted for publication to the Elsevier Journal of the Oceanic Engineering.

Lapierre, L., Soetanto, D., and Pascoal, A. (2003). Adaptive,non-singular path-following of dynamic wheeled robots. In Proc. of the $42^{\text {nd }}$ IEEE Conference on Decision and Control, Maui, Hawaii USA.

Manley, J. (2008). Unmanned Surface Vehicles, 15 years of development. In Proc. of MTS/IEEE Oceans'08.

Manley, J., Marsh, A., Cornforth, W., and Wiseman, C. (2000). Evolution of the autonomous surface craft AutoCat. In Proc. of Oceans'00, volume 1, pages 403-408.

Martins, A., Almeida, J., Silva, E., and Pereira, F. (2006). Vision-based autonomous surface vehicle docking manoeuvre. In Proc. of 7th IFAC Conference on Manoeuvring and Control of Marine Craft, Lisbon, Portugal. 


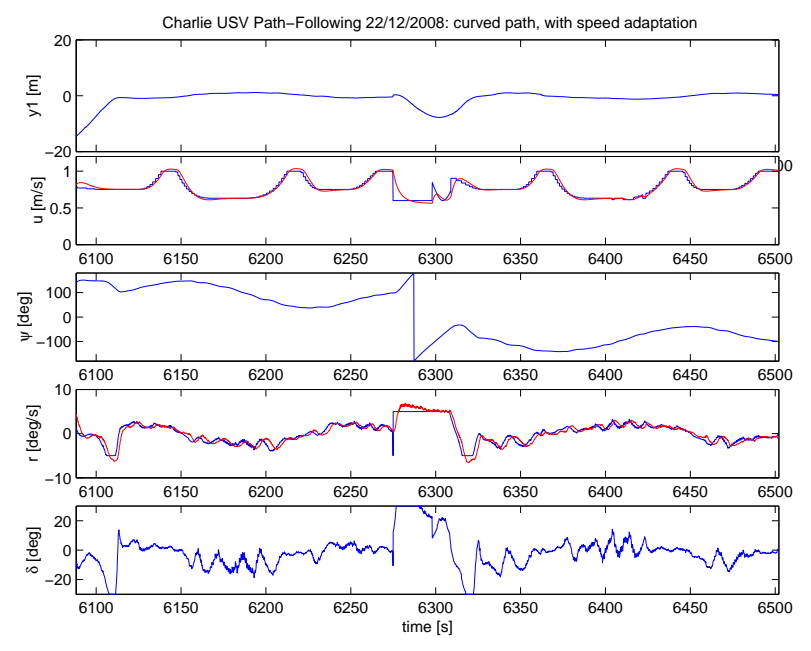

Figure 11: Path-Following: curved path with speed adaptation. Charlie USV path-following and navigation variables. From top to bottom: range from the desired path $y_{1}$, reference and estimated surge $u^{*}$ and $\hat{u}$, estimated heading $\hat{\psi}$, reference and estimated yaw rate $r^{*}$ and $\hat{r}$, rudder angle $\delta$

Micaelli, A. and Samson, C. (1993). Trajectory tracking for unicycletype and twosteering wheels mobile robots. Technical report no. 2097, INRIA, Sophia-Antipolis, France.

Pascoal, A. and et al. (2000). Robotic ocean vehicles for marine science applications: the european asimov project. In Proc. of Oceans 2000.

Pascoal, A., Silvestre, C., and Oliveira, P. (2006). Advances in unmanned marine vehicles, chapter Vehicle and mission control of single and multiple autonomous marine robots, pages 353-386. IEE Control Series.

Xu, T., Chudley, J., and Sutton, R. (2006). Soft computing design of a multi-sensor data fusion system for an unmanned surface vehicle navigation. In Proc. of 7th IFAC Conference on Manoeuvring and Control of Marine Craft. 

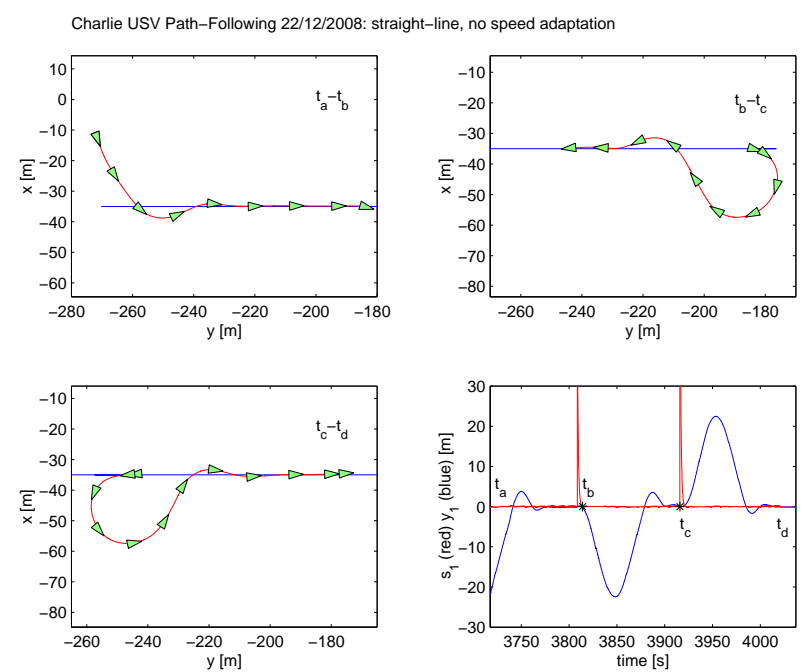

Figure 12: Path-Following: straight line with U-turns without speed adaptation. Charlie USV reference and estimated path: phase 1 (top-left), phase 2 (top-right), phase 3 (bottom-left). Pathfollowing variables (bottom-right): range from the desired path $y_{1}$, virtual target abscissa $s_{1}$.
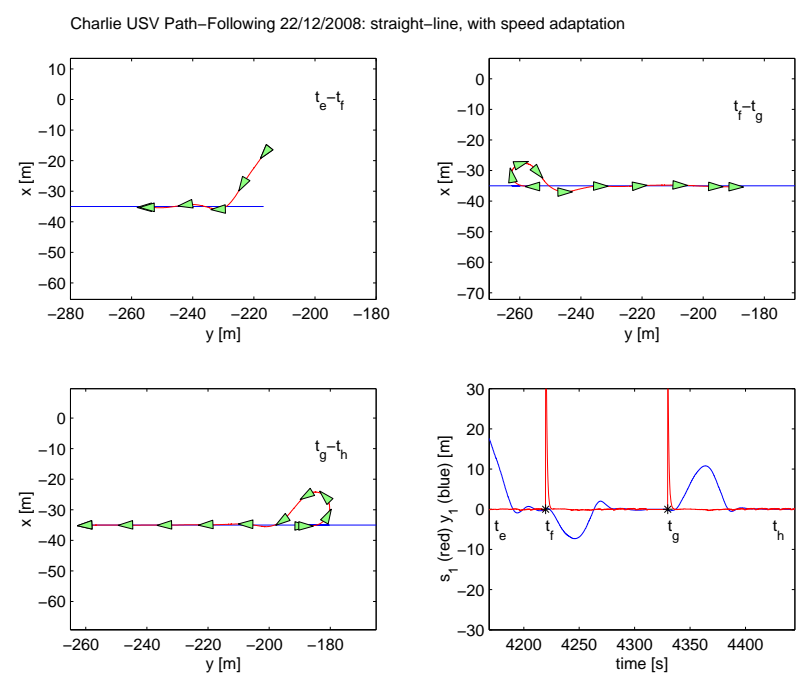

Figure 13: Path-Following: straight line with U-turns with speed adaptation. Charlie USV reference and estimated path: phase 1 (top-left), phase 2 (top-right), phase 3 (bottom-left). Pathfollowing variables (bottom-right): range from the desired path $y_{1}$, virtual target abscissa $s_{1}$. 